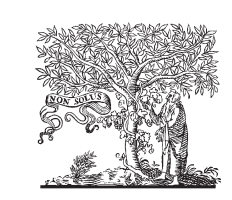

ELSEVIER

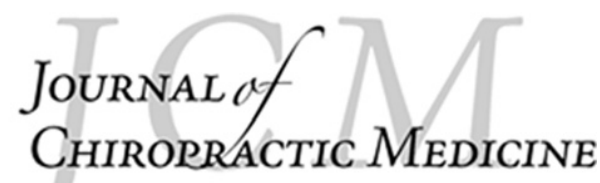

www.journalchiromed.com

\title{
Femoral neck stress fracture in a female athlete: a case report
}

\section{Daniel Avrahami BPHE, DC, MSc ${ }^{a, *}$, Jason A. Pajaczkowski DC, DACRB ${ }^{b}$}

\author{
${ }^{a}$ Chriopractor, Private Practice, Toronto, Ontario, Canada \\ b Assistant Professor, Clinical Education, Canadian Memorial Chiropractic College, Toronto, Ontario, Canada
}

Received 14 February 2012; received in revised form 16 May 2012; accepted 30 May 2012

\section{Key indexing terms:}

Stress fracture;

Femur;

Magnetic resonance imaging;

Chiropractic

\begin{abstract}
Objective: The purpose of this case report is to describe chiropractic rehabilitation of a master's-level athlete with proximal femoral stress fracture and provide a brief discussion of stress fracture pathology.

Clinical Features: A 41-year-old female master's-level endurance athlete presented with chronic groin pain later diagnosed and confirmed by magnetic resonance imaging as a stress fracture of the femoral neck. After diagnosis, the patient was referred to a doctor of chiropractic at week 1 of the non-weight-bearing physical rehabilitation process. At that time, the patient presented with sharp and constant groin pain rated $6 / 10$ on a numeric rating scale.

Intervention and Outcome: This patient avoided weight-bearing activity for 8 weeks while cross-training and was able to return to her sport after this period. The patient was progressed through a series of non-weight-bearing strengthening exercises for the lower extremity. Myofascial release therapy was performed on the gluteal, hip flexor, and groin muscle groups to improve range of motion. Motion palpation testing the lumbar and sacroiliac joints was performed during each session, and manipulative therapy was performed when necessary. The patient was seen once a week for 8 weeks. Reevaluation was performed at week 8 ; at that time, the patient reported no groin pain $(0 / 10)$. The patient was discharged from care and referred back to the supervising physician for clearance to return to sporting activities. One month after discharge, she reported that she was pain free and had fully returned to sport activities.

Conclusion: This case report demonstrates the importance of a through clinical history, physical examination, and magnetic resonance imaging in the accurate diagnosis of a patient with chronic groin pain and that chiropractic care can contribute to rehabilitation programs for these injuries. (C) 2012 National University of Health Sciences.
\end{abstract}

\footnotetext{
* Corresponding author. 390 Bay St., Toronto, Ontario, Canada, M5H 2 Y2.
}

E-mail address: Dr.danavrahami@gmail.com (D. Avrahami). 


\section{Introduction}

Stress fractures can be commonly found in various female subgroups. One of the highest proportions of stress fracture incidence rates in women is found in military personnel. ${ }^{1}$ Another common group of women that have the tendency to develop stress fractures are endurance athletes that perform repetitive weightbearing sports such as distance running. ${ }^{2}$ Stress fractures can also frequently occur in adolescent athletes with amenorrhea, eating disorders, and osteopenia, otherwise known as the female athlete triad. ${ }^{3,4}$

Several locations in the lower extremity have a predisposition towards developing stress fractures, including the tibia, metatarsals, and fibula. ${ }^{5,6}$ Owing to the aforementioned subgroups and frequently reported sites of injury, clinicians will frequently screen athletes in these groups and add a differential diagnosis of stress fracture. However, it is still possible for healthy athletes to sustain stress injuries or fractures that can easily be overlooked.

It is typical for a clinician to manage an athletic patient with conservative therapy before sending them for advanced imaging. Therefore, the misdiagnosis and subsequent mismanagement of an athlete with groin pain may easily occur, compounded by the complexity of the anatomical structures in the hip. Delay in diagnosis and treatment may result in undesired complications and lost time from sport participation. It is important to have imaging evidence in combination with physical examination findings to appropriately manage these problematic patients. The differential diagnosis of femoral neck stress fracture through clinical history and physical examination needs to be confirmed by advanced imaging.

To date, there have been no reports in the scientific literature of chiropractic management or comanagement of a patient with femoral stress fracture. This case report describes chiropractic rehabilitation of a master's-level athlete with proximal femoral stress fracture and provides a brief discussion of stress fracture pathology.

\section{Case report}

A 41-year-old female personal trainer who competed regularly in mountain biking and cross-country skiing competitions presented to 3 doctors of chiropractic and a physical therapist, on separate occasions, with groin pain during weight-bearing activity. She stated that the pain began as a dull ache only present at the end of a session of physical activity. Over the ensuing season, her symptoms became progressively worse to the point where she experienced constant pain with walking.

On each presentation, the patient was treated using multiple modes of therapy, which included soft tissue manipulation, rehabilitative exercises, and acupuncture. Each therapist treated this patient over the course of 4 months with no improvements in symptoms. After 4 failed treatment programs, the patient was seen by a sports physician. The patient was referred for a bone scan that revealed increased uptake in the right proximal femur. It was suspected that the patient had a fatigue stress fracture and was referred for magnetic resonance imaging (MRI). Various MR images were taken including a coronal T2-weighted fat-saturated image and a coronal T1-weighted image that demonstrated increased and decreased signal intensity at the lesser trochanter, respectively (Fig 1). The axial T2weighted fat-saturated image of the right femoral lesser trochanter demonstrated increased signal intensity with a linear, low-signal fracture line with surrounding edema (Fig 2). Her medical physician recommended that she stop all weight-bearing activity for 8 weeks with a reevaluation at that time.

The patient was referred to a doctor of chiropractic at week 1 of the non-weight-bearing physical rehabilitation process. The patient presented with sharp and constant groin pain rated $6 / 10$ on a numeric rating scale. There was no associated weakness or paresthesia reported. The patient did report that her lower extremity

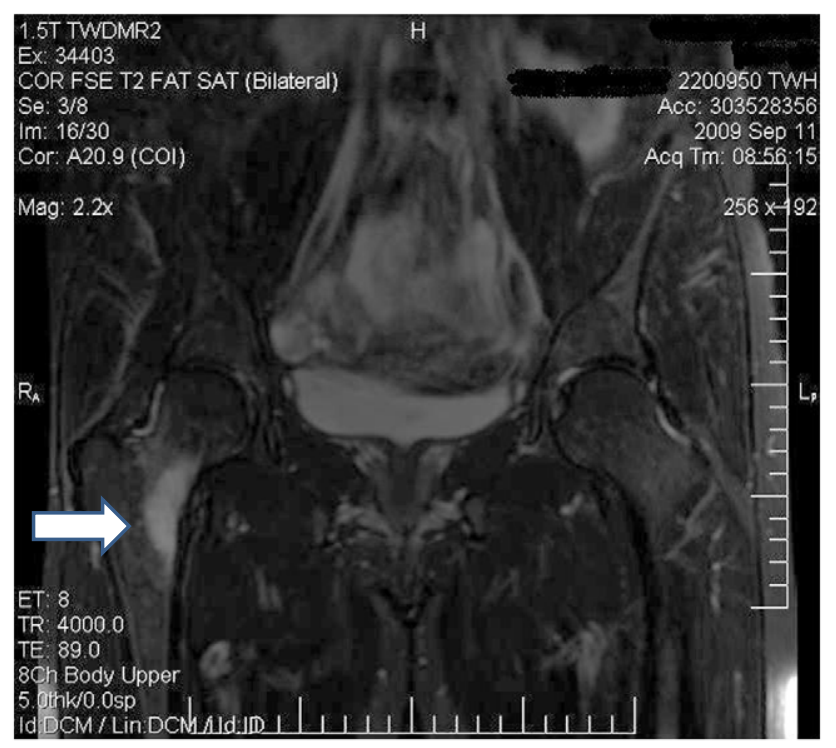

Fig 1. T2 fat-saturated MRI in the coronal plane. There is increased signal intensity at the lesser trochanter of the right femur (arrow). 


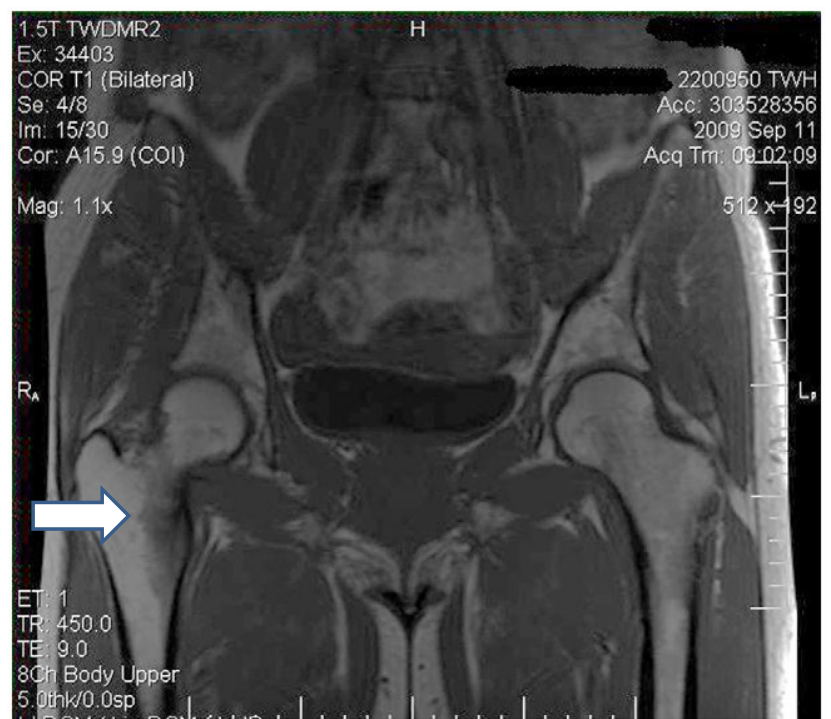

Fig 2. T1-weighted MRI in the coronal plane. There is decreased signal intensity at the lesser trochanter of the right femur (arrow).

strength and endurance were significantly decreased because she has not been able to train or compete in her sports. Neurological evaluation revealed sensory, motor, and reflex testing to be within normal limits. Vibration testing did not aggravate her symptoms. Gait assessment revealed an antalgic gait pattern. Static palpation of this patient revealed pain in the groin region around the hip flexor insertion. Active straight leg raise was painful, and hip flexor muscle strength testing was rated $4 / 5$.

The patient was progressed through a series of nonweight-bearing strengthening exercises for the lower extremity. Myofascial release therapy was performed on the gluteal, hip flexor, and groin muscle groups to ensure adequate range of motion. Motion palpation testing the lumbar and sacroiliac joints was performed during each session, and manipulative therapy was performed when necessary. The patient was seen once a week for 8 weeks.

A reevaluation was performed at week 8 of the nonweight-bearing physical rehabilitation process. At this time, the patient did not have any groin pain, rated $0 / 10$ on the numeric rating scale. There was no associated weakness or paresthesia reported. Results of vibration testing and gait assessment were unremarkable. Static palpation of this patient did not reveal any pain in the groin region or around the hip flexor insertion. Active straight leg raise was asymptomatic, and hip flexor muscle strength testing was rated $5 / 5$. At this point, the patient was discharged from care and referred back to the supervising physician for clearance to return to her sporting activities. The patient reported no adverse reactions to chiropractic care. Through e-mail communication with this patient, 1 month after her discharge from therapy, she stated that she had returned to her full sporting activities pain-free.

\section{Discussion}

\section{Case discussion}

This case describes the findings and chiropractic rehabilitation of a clinically suspected proximal femoral stress fracture in a master's-level athlete confirmed by MRI. This patient avoided weightbearing activity for 8 weeks while cross-training and receiving chiropractic care. The patient was able to return to her sport after this period. Non-weightbearing cross-training exercises in combination with chiropractic care can help expedite the rehabilitation process and allow athletes to return to their sport within an earlier time frame.

\section{Review of stress fracture pathology}

\section{Definition}

Bone stress fractures can be defined as insufficiency or fatigue fractures. When injury resulted from normal stress applied to abnormal bone, an insufficiency fracture occurs. Elderly women with osteoporosis tend to develop these types of injuries; however, any disorder that results in diminished resistance to physical forces as a result of altered osseous health may result in an insufficiency fracture, including but not limited to Paget disease, hyperparathyroidism, rheumatoid arthritis, diabetes, scurvy, osteomalacia, osteogenesis imperfecta or rickets, and malignancy. ${ }^{7,8}$

Conversely, normal bone that is subjected to excessive stress can develop fatigue fractures. ${ }^{9}$ Bone remodels in response to repetitive submaximal stresses. ${ }^{6}$ With increased stress, the bone does not remodel sufficiently to accommodate for the rapid bone breakdown that can eventually lead to microfractures and stress fracture. ${ }^{10}$

\section{Histology}

The histology of fatigue stress fractures shows that repetitive response to stress leads to reparative osteoblastic bone formation activity that is outpaced by osteoclastic breakdown, resulting in temporary weakening of bone. ${ }^{11}$ Trabecular microfractures can 
result if physical activity is continued under these circumstances, and they explain the early bone marrow edema seen on MRI scanning. Reinforcement is created by forming new periosteal bone. However, if the osteoclastic activity continues to exceed the rate of osteoblastic formation, a full cortical break will ensue. ${ }^{6}$

\section{Location}

Generally, there are 3 femoral locations susceptible to stress fractures. The distal shaft, between the distal one-third and the femoral condyles, is a common site. ${ }^{12}$ There is also a relatively high incidence of proximal shaft fractures that occur primarily on the medial surface. ${ }^{13}$ The highest incidence rates of femoral stress fractures occur at the femoral neck. ${ }^{12}$ Kiuru et al ${ }^{14}$ demonstrated that stress fractures in this area and along the proximal shaft of the femur were $50 \%$ more common than stress injuries of the pelvis.

Typically, during running activities, the femoral neck is subjected to large anteriorly and medially oriented shear forces. ${ }^{15}$ Edwards et al ${ }^{15}$ demonstrated that axial forces and moments at the neck were no larger than those experienced by the rest of the femur and occurred during the braking force delivered at heel strike during the gait cycle. The impact phase of running is associated with a high rate of loading, and microdamage to cortical bone increases proportionally to loading rate. ${ }^{16}$ Repetitive activities in conjunction with the small diameter of the femoral neck as compared with the rest of the femur will threaten the integrity of the bone.

It is possible that, in an in vivo situation, the difference between medial surface compressive stress and lateral surface tensile stress outweighs the difference in anisotropic strength. Because cortical bone is strongest in compression, the reason that proximal shaft fractures materialize on the medial surface is unclear. ${ }^{17}$ Edwards et al ${ }^{15}$ suggested that these differences may become more pronounced during excessive training where hip abductor muscles fatigue while resisting the anterior-posterior bending moment.

\section{Etiology}

Fatigue fractures that occur in young individuals are often a result of repetitive athletic activity. Distance runners, dancers, and military recruits are commonly affected and are at an increased risk to developing stress fractures in the hip. ${ }^{18}$ Insufficiency fractures occur in the elderly population with osteoporosis, osteomalacia, rheumatoid arthritis, diabetes, hyperparathyroidism, or radiation therapy. ${ }^{18}$
Stress fractures occur because of increased load after fatigue of supporting structures, contractile muscular forces acting across and on the bone, or a combination of both factors. ${ }^{6}$ The most significant factor in producing a stress fracture is cited as an alteration in an athlete's training program. ${ }^{10} \mathrm{~A}$ rapid change can have a profound effect on an athlete's body. Increasing mileage, pace, volume, or cross-training activity that has been inserted into the program without adequate time for physiologic adaptation to accommodate the new forces can lead to stress fractures. Training surfaces that are too hard or too soft are also important precursors to lower extremity overuse injuries. Failure to follow intensive training days with easy ones also can contribute to injury.

Intrinsic risk factors that contribute to stress fracture injuries include female sex, amenorrhea, lower bone density, inadequate muscle function, and biomechanical features. Extrinsic risk factors that contribute to stress fracture injuries include overtraining, inadequate equipment, and the energetic nutrition deficit. Many patients may present with a coexistence of different risk factors, which makes the isolation of etiologic variables so difficult. ${ }^{5}$

Research has also shown that cortical bone strength, cortical area, and muscle cross-sectional area are all lower in runners with a history of stress fracture. However, the lower strength was appropriate for the smaller muscle size, suggesting that interventions to reduce stress fracture risk might be aimed at improving muscle size and strength. ${ }^{19}$

Inadequate diet and nutrition can also contribute towards the development of stress fractures. Smith ${ }^{20}$ demonstrated an association between bone mineral density and veganism. Vegans tend to have a lower intake of calcium and vitamin $\mathrm{D}$, as well as a lower overall bone mineral density.

\section{Examination}

The clinical diagnosis of bone stress injury is difficult and tends to be unspecific because symptoms are often insidious and pain is diffuse or radiating. ${ }^{21}$ Frequently, the diagnosis of stress fracture is clinically made through a thorough history and physical examination.

The primary presenting symptom of a femoral neck stress fracture is usually anterior groin pain. The pain is often exacerbated by training, such as jogging or running, and is relieved by rest. ${ }^{4}$ On physical examination, the most obvious feature is localized bony tenderness. Sometimes, redness, swelling, or periosteal thickening may be present at the site of the stress fracture. ${ }^{22}$ Other findings include limited range 
of motion of the hip, pain on forced rotation or axial loading, and tenderness over the involved bone. A nonspecific test commonly used is the hop test. If the patient reproduces the pain by hopping on the involved extremity, the result is considered positive. The result of this test will be positive in approximately $70 \%$ of patients with femoral neck stress fractures. ${ }^{23}$ The fulcrum test is another test commonly used to detect femoral shaft stress fractures. ${ }^{24}$

\section{Imaging}

Stress fractures of the femoral neck can result in hip pain, and it is often difficult to locate the origin of pain even with a thorough history and clinical examination. ${ }^{14}$ It is important to prevent complications resulting from delay in diagnosis and treatment of stress fractures of the femoral neck. Therefore, appropriate imaging strategies through evidence-based diagnostic imaging practice guidelines in decision making for the appropriate use of diagnostic imaging of lower extremity disorders are necessary to accurately diagnose stress fractures. ${ }^{25}$ There are various imaging techniques that can be used to confirm the diagnosis.

In the majority of stress fractures, there is no obvious abnormality on plain radiograph. Kiuru et al ${ }^{14}$ demonstrated that radiography is of no diagnostic value in assessment of sacral stress injury, most likely because of the three-dimensional geometry of the sacral alae and the possible overlying bowel gas.

Regarding stress fractures, the role of computed tomography (CT) is limited mainly to differential diagnosis. ${ }^{26}$ The CT scan can be used to visualize the fracture and distinguish between a stress reaction and stress fracture. Computed tomographic scans can demonstrate fracture lines in locations where radiography is not diagnostic. However, the diagnostic value of CT is lower than that of bone scintography and MRI, except in longitudinal stress fractures. ${ }^{27}$

A bone scan is extremely sensitive; but the fracture itself is not visualized, and it may be difficult to precisely locate the site. ${ }^{6}$ The scan will detect stress fractures during remodeling, so the findings must be closely correlated with the clinical picture. The characteristic bone scan appearance of a stress fracture is of a sharply marginated area of increased uptake. ${ }^{6}$

Magnetic resonance imaging is being used with increasing frequency for patients with stress fractures. ${ }^{14}$ Anderson and Greenspan ${ }^{26}$ (1996) claim that MRI is extremely sensitive in detecting early signs of bone stress injury. Short tau inversion recovery and other fat suppression techniques can be used to maximize the sensitivity of MRI and consequently to improve diagnostic accuracy. ${ }^{26}$ The typical findings on MRI are of periosteal and marrow edema, as well as fracture line. ${ }^{22}$ Abnormalities associated with the iliopsoas tendon and its insertion, including marrow edema at the lesser trochanter, periostitis around the lesser trochanter, and bone marrow edemas in the femoral neck, are common findings that may be associated with stress fracture near the lesser trochanter. ${ }^{28}$ Each of these findings in isolation is nonspecific and can occur in both stress reactions and enthesopathies. Therefore, it is important to take these findings with great prudence in the absence of a clear fracture line with imaging, as patient management differs significantly between these 2 diagnoses. ${ }^{28,29}$

Magnetic resonance imaging or bone scintigraphy should be undertaken if the result of radiography is negative. Missing a stress injury of the femoral neck is feared because it can lead, through stress fracture, to a complicated displaced fracture and possibly avascular necrosis of the femoral head associated with displaced fracture of the femoral neck. ${ }^{4,14}$

\section{Intervention}

Treatment depends on the severity and location of the fracture, but often involves long periods of rest followed by a gradual return to activity. It is important to ascertain the appropriate diagnosis in patients suspected with a stress fracture at the femoral neck, as the plan of management can vary if diagnosed with an iliopsoas insertional tendinopathy or enthesopathy. Initial therapy for musculotendinous injuries typically consists of stretching; strengthening exercises; and, in some cases, local corticosteroid injection. ${ }^{28}$ In addition, there are a growing number of peer-reviewed studies of manipulative therapy for lower extremity disorders. ${ }^{30}$ On the other hand, several months of reduced weight-bearing or nonambulatory activities along with close monitoring of patient progress may be necessary before training can be resumed in stress fracture patients. ${ }^{24,30}$

Operative fixation is not frequently required in young athletes unless progression of pain or fracture is observed. Full recovery can be made after this rest period, and the patient may return to his or her original activity safely. Adequate diet and nutrition, especially in the young athlete, must be incorporated into the treatment plan. ${ }^{31}$

\section{Prevention}

Clinicians should realize that the process of a stress fracture is on a continuum both physiologically and clinically. It is important to catch these cases early to accelerate rapid healing. Therefore, it is ideal to 
intercept the patient during the stress reaction phase prior to the patient developing a stress fracture in which a distinct fracture line is visible on imaging.

Treatment of stress fractures revolves around rest from the aggravating activity. If treatment is prompt and the athlete adheres to activity modification, he or she can usually return to sport with no complications within 6 to 8 weeks. ${ }^{22}$ Return to activity should be based on the athlete's symptoms and physical findings. Once the athlete is pain-free, the sport can be resumed at a slow progression. One of the most important aspects of the rehabilitation process involves maintaining fitness levels during the healing process. Examples of non-weight-bearing cross-training sports include cycling, swimming, upper body weights, and water running. ${ }^{22}$ Management of stress fractures also involves identification of the factors that have contributed to the injury. Once the culprit has been identified, correction and modification of these factors will reduce the risk of the injury recurring. ${ }^{22}$

Nutritional preventative measures should also be considered for individuals who are thought to be at risk for developing stress fractures. Individuals with low calcium and vitamin D levels will tend to have lower bone mineral density overall. The addition of calciumfortified foods (1300 mg/d of calcium) and extreme dietary changes along with sunlight and exercise may provide enough calcium to a growing adolescent without the addition of dairy. ${ }^{32}$

Muscular strength, reactivity, and endurance can also play an important role in preventing stress fractures in the hip. Typically, the torque imposed on the medial aspect of the hip joint is counteracted by the gluteus medius and minimus muscles. ${ }^{18}$ These stresses are secondary to compression and bending. If the gluteal muscles are fatigued, their counterbalancing effects are minimized, and muscle fatigue is thought to result in more load transmission to bone, thereby increasing the risk of osseous injury. ${ }^{33}$ Therefore, the gluteus medius and minimus must be strong, reactive, and resistant to fatigue to avoid undue stress imparted on the femur. ${ }^{18}$

It has also been postulated that, during repetitive athletic activity, chronic traction forces from the iliopsoas muscle can put stress on the femoral neck and increase axial bending strain on the medial aspect of the femoral neck. ${ }^{34,35}$ The flexor musculotendinous unit acts as a shock-absorbing spring during running. ${ }^{36,37}$ Because musculotendinous units are prone to injury, the iliopsoas musculotendinous unit can be injured by repetitive, excessive, or unbalanced contraction of the iliopsoas muscle during running. ${ }^{33}$ Muscular injury can expose the femoral neck to increased forces and reactive marrow edema at the lesser trochanter, and place forces at the femoral neck that expose this site to stress injury. Therefore, proper training programs and adequate rest are imperative to avoiding these problems in repetitive sport athletes.

\section{Limitation}

During the reevaluation examination at 8 weeks, no imaging was taken. Without this imaging, we are unable to confirm that there was complete healing at the femoral neck. In addition, we are not able to compare the original images with the condition of the femoral neck at the time the patient was discharged. Finally, after the patient was discharged, she was only contacted once via e-mail. A follow-up within the clinic at 1 month, 3 months, and 1 year would have been prudent for such a case.

As with all case reports, causation cannot be determined. It is possible that other factors were responsible for the patient's response or that she may have responded regardless of care. The findings in this case may not necessarily be generalizable to similar patients; each patient's case must be evaluated and managed according to that patient's findings and needs.

\section{Conclusion}

This case summarizes the literature on repetitive stress fractures and provides insight into the importance of early detection through clinical history and physical examination, which can be confirmed by MRI. This case demonstrates the importance of the non-weight-bearing rehabilitation therapy process that can be supplemented with chiropractic care. Ascertaining the appropriate diagnosis and providing the necessary management for these patients are crucial to their rehabilitation process and helping them return to their sport.

\section{Funding sources and potential conflicts of interest}

No funding sources or conflicts of interest were reported for this study.

\section{References}

1. Niva MH, Mattila VM, Kiuru MJ, Pihlajamaki HK. Bone stress injuries are common in female military trainees: a preliminary study. Clin Orthop Relat Res 2009;467:2962-9. 
2. Williams TR, Puckett ML, Denison G, Shin AY, Gorman JD. Acetabular stress fractures in military endurance athletes and recruits: incidence and MRI and scintigraphic findings. Skeletal Radiol 2002;31:277-81.

3. Feingold D, Hame SL. Female athlete triad and stress fractures. Orthop Clin North Am 2006;37:575-83.

4. Okamoto S, Arai Y, Hara K, Tsuzihara T, Kubo T. A displaced stress fracture of the femoral neck in an adolescent female distance runner with female athlete triad: a case report. Sports Med Arthrosc Rehabil Ther Technol 2010;2:6.

5. Ben Chihaoui M, Elleuch M, Sahli H, Cheour I, Romdhane RH, Sellami S. Stress facture: epidemiology, physiopathology and risk factors. Tunis Med 2008;86:1031-5.

6. Fredericson M, Jennings F, Beaulieu C, Matheson GO. Stress fractures in athletes. Top Magn Reson Imaging 2006;17: 309-25.

7. Pentecost RL, Murray RA, Brindley HH. Fatigue, Insufficiency, and Pathologic Fractures. JAMA 1964;187:1001-4.

8. Markey KL. Stress fractures. Clin Sports Med 1987;6:405-25.

9. Belkin SC. Stress fractures in athletes. Orthop Clin North Am 1980;11:735-41.

10. Jones BH, Harris JM, Vinh TN, Rubin C. Exercise-induced stress fractures and stress reactions of bone: epidemiology, etiology, and classification. Exerc Sport Sci Rev 1989;17:379-422.

11. Li GP, Zhang SD, Chen G, Chen H, Wang AM. Radiographic and histologic analyses of stress fracture in rabbit tibias. Am J Sports Med 1985;13:285-94

12. Niva MH, Kiuru MJ, Haataja R, Pihlajamaki HK. Fatigue injuries of the femur. J Bone Joint Surg Br 2005;87:1385-90.

13. Korpelainen R, Orava S, Karpakka J, Siira P, Hulkko A. Risk factors for recurrent stress fractures in athletes. Am J Sports Med 2001;29:304-10.

14. Kiuru MJ, Pihlajamaki HK, Ahovuo JA. Fatigue stress injuries of the pelvic bones and proximal femur: evaluation with MR imaging. Eur Radiol 2003;13:605-11.

15. Edwards WB, Gillette JC, Thomas JM, Derrick TR. Internal femoral forces and moments during running: implications for stress fracture development. Clin Biomech (Bristol, Avon) 2008;23:1269-78.

16. Schaffler MB, Radin EL, Burr DB. Mechanical and morphological effects of strain rate on fatigue of compact bone. Bone 1989;10:207-14.

17. Reilly DT, Burstein AH. The elastic and ultimate properties of compact bone tissue. J Biomech 1975;8:393-405.

18. Egol KA, Koval KJ, Kummer F, Frankel VH. Stress fractures of the femoral neck. Clin Orthop Relat Res 1998:72-8.

19. Popp KL, Hughes JM, Smock AJ, et al. Bone geometry, strength, and muscle size in runners with a history of stress fracture. Med Sci Sports Exerc 2009;41:2145-50.

20. Smith AM. Veganism and osteoporosis: a review of the current literature. Int J Nurs Pract 2006;12:302-6.
21. Shin AY, Morin WD, Gorman JD, Jones SB, Lapinsky AS. The superiority of magnetic resonance imaging in differentiating the cause of hip pain in endurance athletes. Am J Sports Med 1996;24:168-76.

22. Brukner P, Bennell K. Stress fractures in female athletes. Diagnosis, management and rehabilitation. Sports Med 1997;24:419-29.

23. Clement DB, Ammann W, Taunton JE, et al. Exercise-induced stress injuries to the femur. Int J Sports Med 1993;14:347-52.

24. Johnson AW, Weiss Jr CB, Wheeler DL. Stress fractures of the femoral shaft in athletes-more common than expected. A new clinical test. Am J Sports Med 1994;22:248-56.

25. Bussières AE, Taylor JA, Peterson C. Diagnostic imaging practice guidelines for musculoskeletal complaints in adults-an evidence-based approach. Part 1. Lower extremity disorders. J Manipulative Physiol Ther 2007;30(9):684-717.

26. Anderson MW, Greenspan A. Stress fractures. Radiology 1996;199:1-12.

27. Feydy A, Drape J, Beret E, et al. Longitudinal stress fractures of the tibia: comparative study of CT and MR imaging. Eur Radiol 1998;8:598-602.

28. Nguyen JT, Peterson JS, Biswal S, Beaulieu CF, Fredericson M. Stress-related injuries around the lesser trochanter in longdistance runners. AJR Am J Roentgenol 2008;190:1616-20.

29. Adler RS, Buly R, Ambrose R, Sculco T. Diagnostic and therapeutic use of sonography-guided iliopsoas peritendinous injections. AJR Am J Roentgenol 2005;185:940-3.

30. Brantingham JW, Globe G, Pollard H, Hicks M, Korporaal C, Hoskins W. Manipulative therapy for lower extremity conditions: expansion of literature review. J Manipulative Physiol Ther 2009;32(1):53-71.

31. Ivkovic A, Bojanic I, Pecina M. Stress fractures of the femoral shaft in athletes: a new treatment algorithm. Br J Sports Med 2006;40:518-20.

32. Ross K, Fahey M. Bilateral femoral supracondylar stress fractures in a cross country runner. Orthopedics 2008;31:803.

33. Gao X, Wilde PE, Lichtenstein AH, Tucker KL. Meeting adequate intake for dietary calcium without dairy foods in adolescents aged 9 to 18 years (National Health and Nutrition Examination Survey 2001-2002). J Am Diet Assoc 2006;106:1759-65.

34. Moore JS. Function, structure, and responses of components of the muscle-tendon unit. Occup Med 1992;7:713-40.

35. Stevens MA, El Khoury GY, Kathol MH, Brandser EA, Chow S. Imaging features of avulsion injuries. Radiographics 1999;19:655-72.

36. Simoes JA, Vaz MA, Blatcher S, Taylor M. Influence of head constraint and muscle forces on the strain distribution within the intact femur. Med Eng Phys 2000;22:453-9.

37. Arendt EA. Stress fractures and the female athlete. Clin Orthop Relat Res 2000:131-8. 